\title{
Human Cardiac Stem Cells
}

\author{
A. Leri*, S. Cascapera *, C. Bearzi*, J. Kajstura*, R. Bolli** and P. Anversa* \\ *Cardiovascular Research Institute, New York Medical College, Valhalla, New York, 10595; \\ **Division of Cardiology, University of Louisville, Louisville, Kentucky, 40292
}

The engrained view of the heart as a post-mitotic organ has been challenged by recent observations in which a cardiac stem cell (CSC) compartment has been identified in rodents [1-3]. When injected locally, these progenitor cells differentiate into myocytes and coronary vessels regenerating dead, infarcted myocardium [1]. However, the ability of these cells to reconstitute the damaged heart has been controversial [2] and questions have been raised concerning the actual existence of resident progenitor cells in the human heart [4] and the potential therapeutic efficacy of the CSC pool in humans. Because of the clinical needs of replacing lost or poorly contracting myocytes with new, better functioning cells, the hemopoietic and non-hemopoietic fractions of the bone marrow have been utilized as a source for regeneration of myocytes and coronary vessels in the diseased heart $[5,6]$. However, differentiation of bone marrow cells into cells different from those of the organ of origin is a complex process that requires a reprogramming of the intrinsic commitment of the primitive cells with the acquisition of the adult phenotype of the cells of the host tissue [7]. Therefore, the documentation of resident stem cells in the human heart is of great significance, biologically and clinically. These cells would control cell turnover and the continuous formation of fully developed myocytes and coronary vessels, rapidly and efficiently, without a switch in cell fate.

We report that we have identified a human CSC. Effort was made to establish the conditions for the isolation and expansion of potential progenitor cells from small samples of human myocardium. By this approach, we have identified a c-kit positive cardiac stem cell that is self-renewing, clonogenic and multipotent. This primitive cell has the morphological properties and expresses some of the surface antigens commonly found in mesenchymal stem cells. Additionally, when locally injected in the infarcted myocardium of immunodeficient rats and mice, cardiac stem cells regenerate myocytes and coronary vessels. The culture of cardiac stem cells, from their outgrowth from the myocardial samples to their implantation in infarcted animals, was achieved in the absence of animal products.

Traditionally the heart has been viewed as a static organ incapable of repairing any form of damage. According to this paradigm, the number of myocytes is established at birth [8] and this population of terminally differentiated myocytes is irreplaceable throughout the life of the organ and the organism. Myocyte aging is dictated by the age of the organism and myocytes can only increase in size or die. In agreement with this persistent notion of the heart, parenchymal cells are permanently lost and there is no reserve mechanism that regulates myocyte death and the wear and tear resulting from the physiological demands dictated by daily life. In theory, myocytes can live more than 100 years in humans and only the intracellular organelles are continuously replaced leaving intact the viability of the cells. The demonstration that a subpopulation of small amplifying myocytes can divide acutely and chronically after infarction $[9,10]$ or pressure overload [11] in humans has been vigorously challenged $[8,12]$ and criticisms varied from technical errors in the identification of replicating cells $[13,14]$ to the lack of relevance of the findings [15]. In spite of irrefutable lines of evidence of a highly 
significant regeneration of myocytes in the adult human heart [9-11], the criticisms persisted and the concept of the heart as a post-mitotic organ is still widely accepted in the scientific community [15].

The recognition that the mammalian heart possesses a stem cell compartment [1-3] that can regenerate myocytes and coronary vessels [1] raises the unique possibility to reconstitute dead myocardium after infarction, to repopulate the hypertrophic decompensated heart with new better functioning myocytes and vascular structures and perhaps to reverse ventricular dilation and wall thinning, restoring the physiological and anatomical characteristics of the normal heart. This notion is supported here by the identification of resident CSCs in the human heart and by the demonstration that human CSCs can be isolated from small tissue fragments and expanded in vitro. Additionally, the local injection of CSCs in infarcted immunodeficient animals repairs the necrotic myocardium by forming functionally competent myocytes, coronary arterioles and capillary profiles. These observations have changed the perennial view of the heart as a post-mitotic organ [15] and have formed the basis of a new paradigm in which multipotent CSCs are implicated in the normal turnover of myocytes, endothelial cells and smooth muscle cells in the adult myocardium. The heart is a dynamic organ constantly renewing its cell populations. Moreover, the new paradigm introduces a more biologically interesting model of cardiac growth, aging and death. The human heart belongs to the group of self-renewing organs such as the bone marrow, the skin, the brain and the intestine.

\section{References}

[1] Beltrami, A.P. et al. Cell 114, 763 (2003).

[2] Oh H. et al. Proc. Natl. Acad. Sci. USA 100, 12313 (2003).

[3] Matsuura K. et al. J. Biol. Chem. 279, 11384 (2004).

[4] A.J. Wagers and I.L. Weissman. Cell 116, 639 (2004).

[5] N. Rosenthal. N. Engl. J. Med. 349, 267 (2003).

[6] Mathur A. and J.F. Martin. Lancet 364, 183 (2004).

[7] Tosh D. and Slack J.M.. Nat. Rev. Mol. Cell. Biol. 3, 187 (2004).

[8] MacLellan, W.R. \& Schneider, M.D. Annu. Rev. Physiol. 62, 289-319 (2000).

[9] Beltrami, A.P. et al. N. Engl. J. Med. 344, 1750-1757 (2001).

[10] Kajstura, J. et al. Proc. Natl. Acad. Sci. USA 95, 8801-8805 (1998).

[11] Urbanek, K. et al. Proc. Natl. Acad. Sci. USA 100, 10440-10445 (2003).

[12] Chien, K.R. Nature 428, 607-608 (2004).

[13] Murry, C.E. et al. Nature 428, 664-668 (2004).

[14] Balsam, L.B. et al. Nature 428, 668-673 (2004).

[15] Nakamura, T. \& Schneider, M.D. Circulation 107, 2638-2639 (2003).

[16] This work was supported by grants from the NIH. 